\title{
Lacosamide and myoclonic seizures: what is the risk of aggravation?
}

\author{
Magdalena Bosak ${ }^{1}$, Kamil Wężyk ${ }^{2}$, Agnieszka Słowik ${ }^{1}$ \\ ${ }^{1}$ Collegium Medicum, Jagiellonian University of Krakow, Krakow, Poland \\ ${ }^{2}$ University Hospital in Krakow, Poland
}

Key words: lacosamide, myoclonic seizures, aggravation, exacerbation

(Neurol Neurochir Pol 2021; 55 (1): 107-109)

\section{To the Editors:}

Lacosamide (LCM), a novel antiseizure medication (ASM), is believed to exert its anticonvulsant action through selective enhancement of slow inactivation of voltage-gated sodium channels.

LCM is indicated for the treatment of focal seizures, and has recently been demonstrated to be efficacious as an adjunctive therapy for primary generalised tonic-clonic seizures (GTCS) [1]. Genetic generalised epilepsies (GGEs) comprise $15-20 \%$ of all epilepsies, and are usually easy to treat with broad-spectrum ASM [2, 3]. Myoclonic and absence seizures in GGEs seem to be more vulnerable to aggravation by some ASMs. The older sodium channel blockers (SCBs) such as phenytoin (PHT), carbamazepine (CBZ), oxcarbazepine (OCBZ), and lamotrigine (LTG), may worsen myoclonic seizures [2].

Here we report a case series of patients who experienced an exacerbation of generalised myoclonic seizures following initiation of LCM in light of the existing literature.

\section{Case 1}

A 47-year-old woman presented to the epilepsy clinic with an increased number of seizures. Her epilepsy had started with myoclonic jerks in the morning at age 13. She had been successfully treated for five years with phenobarbital (PB), then was switched to CBZ for unknown reasons. On CBZ, she experienced absence and myoclonic seizures once per week and generalised tonic-clonic seizures (GTCS) every few years. LCM was added to CBZ three years ago, with a deterioration in seizure control. On LCM $350 \mathrm{mg} /$ day $(150 \mathrm{mg}$ in the morning, $200 \mathrm{mg}$ at night) and CBZ-CR 1,000 mg/day she had daily myoclonic seizures and up to three GTCSs per month. Electroencephalography (EEG) showed background slowing with beta waves excess (she took alprazolam) and generalised polyspike and slow wave discharges (GPSWD) (Fig. 1A). Juvenile myoclonic epilepsy (JME) was diagnosed, LCM had been gradually discontinued, and valproate (VPA) was initiated. On the subsequent visit, while taking CBZ-CR 1,000 mg/day and VPA $1,500 \mathrm{mg} /$ day, she reported no absences, myoclonus or GTCS. EEG revealed GPSWD with normal background (Supp. Fig. 1). CBZ had been gradually withdrawn. EEG taken seven months later on VPA 1,500 mg/day showed normal background without epileptiform discharges (Supp. Fig. 2). The patient was seizure free.

\section{Case 2}

A 34-year-old woman presented with a history of refractory JME that had begun at age 14. She had been treated with VPA, LTG, LEV, PB, topiramate (TPM), but without ever achieving seizure control. On VPA 1,000 mg/day, LTG $150 \mathrm{mg} /$ day, and TPM $400 \mathrm{mg}$ /day she experienced GTCS bimonthly and myoclonic seizures once per week. Because she planned pregnancy, VPA and TPM were gradually withdrawn and LEV 2,000 mg/ day introduced, with no apparent change in seizure control. She delivered a healthy baby by caesarean section. Given its confirmed efficacy as an add-on treatment of primary GTCS, LCM was added to the treatment regimen (LTG $150 \mathrm{mg}$ /day, LEV 2,000 mg/day). Two weeks after initiating treatment with LCM, the frequency of myoclonic seizures increased, reaching one or two every day while taking $100 \mathrm{mg}$ BID. 


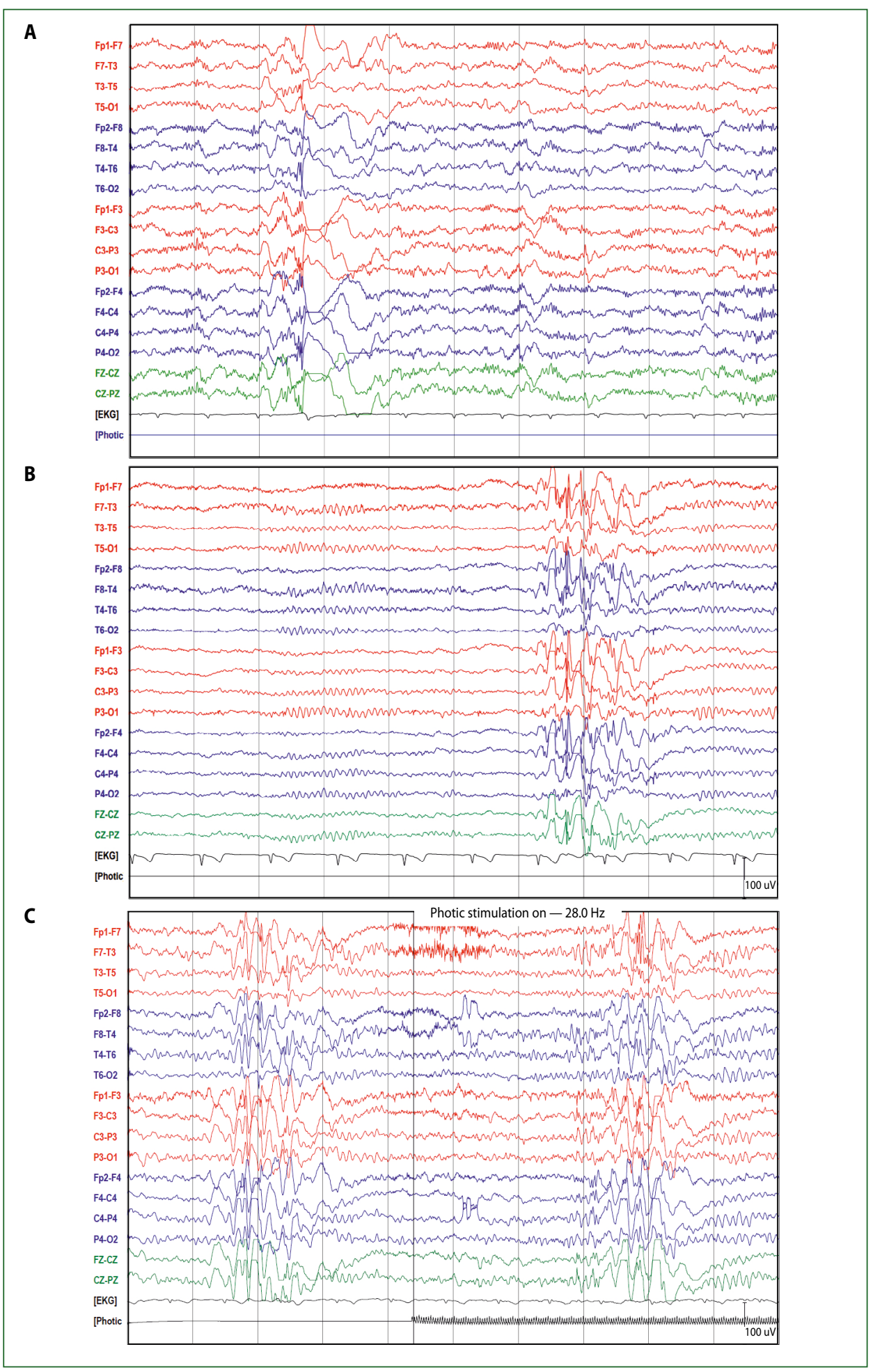

Figure 1. EEG in patients with myoclonic seizures exacerbation on lacosamide. A. Patient 1: background slowing with beta waves excess and polyspike and slow wave discharges; patient had experienced three generalised tonic-clonic seizures on previous day; B. Patient 2: polyspike and wave discharges in drowsiness; C. Patient 3: multiple polyspike and wave discharges during photostimulation 
EEG revealed multiple GPSWD enhanced by sleep (Fig. 1B). The frequency of GTCS did not change. LCM was discontinued after two months and myoclonus frequency decreased significantly to once per week. Follow-up EEG showed no epileptiform discharges (Supp. Fig. 3).

\section{Case 3}

This 27-year-old woman with juvenile absence epilepsy (JAE) had unremarkable family and medical history. At age 13 , she started having weekly absence seizures and occasional GTCS. She continued to have seizures on CBZ. TPM controlled only GTCS and caused cognitive side effects. At age 21 , she was started on VPA 1,000 mg/day, with complete seizure remission. EEG showed no epileptiform discharges (Supp. Fig. 4). Seven years later, the patient was planning pregnancy. VPA was discontinued and the patient was switched to LTG and LEV. On LTG and LEV in mono- and polytherapy she experienced monthly GTCS and intolerable side effects. LEV and LTG were gradually withdrawn, and treatment with LCZ started at a dose of $50 \mathrm{mg} /$ day, increased by $50 \mathrm{mg}$ each week to $100 \mathrm{mg}$ BID, and continued for one year. On LCZ, she experienced a relapse of daily absences and a new occurrence of weekly myoclonic seizures, with no deterioration in GTCS control. EEG revealed multiple GPSWD enhanced by photostimulation and hyperventilation (Supp Fig. 3B). Discontinuation of LCZ and the introduction of low doses of LTG and LEV restored the patient to her previous clinical condition. Absences, myoclonic seizures and epileptiform discharges on EEG resolved, with GTCS occurring once per month (Supp. Fig. 5).

We identified six articles regarding myoclonus being made worse by LCM in the literature. A woman with refractory JME who was started on LCM experienced a subsequent increase of myoclonic seizures, and myoclonus resolved after LCM withdrawal [4]. Another study reported the case of a patient with GTCS who experienced a new occurrence of myoclonic seizures after switching from VPA to LCM. Myoclonic seizures stopped after LCM withdrawal [5]. Italian authors described a young female with focal epilepsy due to focal cortical dysplasia. The addition of LCM to CBZ resulted in an occurrence of epileptic negative myoclonus, which resolved after CBZ dose reduction without any change in LCM dosage [6]. In a case series of nine patients with refractory GTCS, one patient with JAE experienced absence and myoclonic status epilepticus after the introduction of LCM [7]. In an open label study assessing the safety of LCM in the treatment of uncontrolled primary GTCS, one patient reported worsening of myoclonic seizures while taking LCM; this resolved without LCM dosage change [8]. In the study by Vossler et al. among patients with myoclonic seizures, $8.5 \%$ on LCM had a $\geq 50 \%$ increase in myoclonus. Furthermore, one patient had a new occurrence of myoclonic seizures [1].

In total, we have identified 14 cases of myoclonus aggravation or induction: 11 cases were extracted from the literature, and three patients were seen at our centre. All but one suffered from generalised epilepsy. Additionally, some patients experienced GTCS or absence worsening and one suffered from status epilepticus. However, it should be pointed out that in our three cases LCM was added to other SCBs (LTG or CBZ), and the exacerbation of myoclonus may have been synergised/ facilitated by the co-medication with two SCBs.

Our report suggests that LCM may have the potential to worsen or to induce fresh myoclonic seizures. It should be used with caution in GGEs with multiple seizure types. Further studies are needed to evaluate the risk of myoclonus aggravation/induction with lacosamide.

\section{References}

1. Vossler DG, Knake S, O'Brien TJ, et al. SP0982 trial investigators. Efficacy and safety of adjunctive lacosamide in the treatment of primary generalised tonic-clonic seizures: a double-blind, randomised, placebo-controlled trial. J Neurol Neurosurg Psychiatry. 2020; 91(10): 1067-1075, doi: 10.1136/jnnp-2020-323524, indexed in Pubmed: 32817358.

2. Chaves J, Sander JW. Seizure aggravation in idiopathic generalized epilepsies. Epilepsia. 2005; 46 Suppl 9: 133-139, doi: 10.1111/j.1528-1167.2005.00325.x, indexed in Pubmed: 16302887.

3. Wężyk K, Słowik A, Bosak M. Predictors of remission in patients with epilepsy. Neurol Neurochir Pol. 2020; 54(5): 434-439, doi: 10.5603/ PJNNS.a2020.0059, indexed in Pubmed: 32757204.

4. Swaminathan A, Kapoor S. A case report of exacerbation of myoclonus in idiopathic generalized epilepsy from use of lacosamide. Neurology. 2015; 84 (14 Suppl.) Abstracts: AAN 67th Annual Meeting.

5. Birnbaum D, Koubeissi M. Unmasking of myoclonus by lacosamide in generalized epilepsy. Epilepsy Behav Case Rep. 2017; 7: 28-30, doi: 10.1016/j.ebcr.2016.09.006, indexed in Pubmed: 28239547.

6. Belcastro V, Arnaboldi M, Taborelli A, et al. Induction of epileptic negative myoclonus by addition of lacosamide to carbamazepine. Epilepsy Behav. 2011; 20(3): 589-590, doi: 10.1016/j.yebeh.2011.01.022, indexed in Pubmed: 21367668.

7. Abarrategui B, García-García ME, Toledano R, et al. Lacosamide for refractory generalized tonic-clonic seizures of non-focal origin in clinical practice: A clinical and VEEG study. Epilepsy Behav Case Rep. 2017; 8: 63-65, doi: 10.1016/j.ebcr.2017.08.001, indexed in Pubmed: 28948142.

8. Wechsler RT, Yates SL, Messenheimer J, et al. Lacosamide for uncontrolled primary generalized tonic-clonic seizures: An open-label pilot study with 59-week extension. Epilepsy Res. 2017; 130: 1320, doi: 10.1016/j.eplepsyres.2016.12.015, indexed in Pubmed: 28086164. 